\title{
Promoción de Las Habilidades Investigativas Aplicadas a la Docencia en el Magíster en Educación de la Universidad Adventista de Chile: Un Enfoque Estratégico
}

\section{Promotion of Research Skills Applied to Teaching in the Master's Degree in Education at the Adventist Univerty of Chile: A Strategic Aproach}

\section{Promoção de Habilidades de Pesquisa Aplicada ao Ensino do Mestre em Educação na Universidade Adventista do Chile: Uma Abordagem Estratégica}

\author{
Rodrigo Sobarzo-Ruiz (rodrigosobarzo@unach.cl) \\ Universidad Adventista de Chile \\ José Lárez-Hernández (joselarez@ unach.cl) \\ Universidad Adventista de Chile
}

Resumen: El presente estudio tiene por propósito diseñar una propuesta curricular desde un enfoque estratégico, orientada a fortalecer el desarrollo y consolidación de las principales habilidades investigativas, a través de la articulación del eje metodológico en los cursos de Metodología y Seminario de Proyectos de Intervención en Educación y Trabajo Final de Posgrado I y II del Magister en Educación de la Universidad Adventista de Chile. Metodología: Este estudio puede tipificarse como una investigación de tipo Proyecto Factible, sustentado en una investigación documental desarrollada bajo un diseño de tipo bibliográfico. Resultados: Es perentorio reforzar los elementos relacionados con el desarrollo de las habilidades investigativas en las cuatro Unidades Curriculares consideradas como parte del eje metodológico. Dicho fortalecimiento debe realizarse desde la perspectiva de su explicitación en el perfil de competencias para una mayor visualización de éstas y también, en la adecuación en lo atinente al alcance, organización y secuenciación de los núcleos temáticos, los resultados de aprendizaje, la adecuación de las estrategias metodológicas y de evaluación que se empleen. Conclusiones: El desarrollo de habilidades para la ubicación, selección, procesamiento y uso de información de calidad en el abordaje de situaciones problematizadas, a través de los procesos de intervención es fundamental, para contribuir en consolidar el carácter profesionalizante del magister

Palabras-clave: habilidades investigativas; docencia; magister en educación; enfoque estratégico.

Recebido em: 30/05/2021

Aceite em: 13/08/2021 
Abstract: The purpose of this study is: To design a curricular proposal from a strategic approach, oriented to strengthen the development and consolidation of the main research skills through the articulation of the methodological axis in the courses of Methodology and Seminar of Intervention Projects in Education and Postgraduate Final Project I and II of the Master's Degree in Education of the Adventist University of Chile. Methodology: This study can be typified as a feasible project type research, supported by a documentary research developed under a bibliographic type design. Results: It is urgent to reinforce the elements related to the development of research skills in the four curricular units considered as part of the methodological axis. Such strengthening should be carried out from the perspective of its explicitness in the profile of competences for a greater visualization of these and also, in the adequacy regarding the scope, organization and sequencing of the thematic cores, the learning outcomes, the adequacy of the methodological and evaluation strategies to be used. Conclusions: The development of skills for the location, selection, processing and use of quality information in the approach of problematized situations, through the intervention processes is essential to contribute to consolidate the professionalizing character of the master's degree.

Keywords: research skills; teaching; master's degree in education; strategic approach.

Resumo: O objetivo deste estudo é: elaborar uma proposta curricular a partir de uma abordagem estratégica, orientada a fortalecer o desenvolvimento e consolidação das principais competências de pesquisa através da articulação do eixo metodológico nos cursos de Metodologia e Seminário de Projetos de Intervenção em Educação e Projeto Final de Pós-graduação I e II do Mestrado em Educação da Universidade Adventista do Chile. Metodologia: Este estudo pode ser tipificado como uma pesquisa do tipo projeto viável, baseado em uma pesquisa documental desenvolvida sob um desenho de tipo bibliográfico. Resultados: É urgente reforçar os elementos relacionados ao desenvolvimento da capacidade de pesquisa nas quatro unidades curriculares consideradas como parte do eixo metodológico. Tal fortalecimento deve ser realizado a partir da perspectiva de sua explicitação no perfil das competências para uma maior visualização das mesmas e também, na adequação quanto ao escopo, organização e sequenciamento dos núcleos temáticos, os resultados da aprendizagem, a adequação das estratégias metodológicas e de avaliação utilizadas. Conclusões: O desenvolvimento de habilidades para a localização, seleção, processamento e utilização de informações de qualidade na abordagem de situações problematizadas, através dos processos de intervenção é fundamental, para contribuir em consolidar o caráter profissionalizante do mestrado.

Palavras-chave: habilidades de pesquisa; ensino; mestrado em educação; foco estratégico.

\section{INTRODUCIÓN}

Recebido em: 30/05/2021

Aceite em: 13/08/2021 
EL desarrollo de habilidades investigativas, constituye sin lugar a dudas un elemento de particular importancia para la formación profesional del profesorado, pues a través de ellas, el docente adquiere un conjunto de herramientas heurísticas para el abordaje, comprensión y transformación de la realidad en la cual le ha tocado desenvolverse.

Desde esta perspectiva la formación en posgrado es un desafío para cualquier casa de estudio, siendo el foco principal que los futuros magister, sean capaces de pensar y repensar desde una postura críticamente crítica reflexiva su quehacer profesional. Ello demanda obligatoriamente el dominio conceptual e instrumental de elementos asociados a los procesos de investigación, para la generación de soluciones a las problemáticas propias de su especialidad en el marco de su formación disciplinar recibida. Es por ello quepor lo que, desde la Universidades se generan distintas ofertas académicas que colocan a disposición de los interesados diversos programas de estudio, orientados al desarrollo de competencias investigativas, o a la profundización en aspectos del conocimiento especializado con fines de aplicación, siendo estos últimos los que se encuentran encaminados a la formulación de proyectos de intervención para la solución de problemáticas del medio laboral, por su clara orientación profesional.

El estudiante de los programas de posgrado profesionales idealmente debería integrar un conjunto de elementos de orden conceptual, procedimental y actitudinal que le permitirán actuar de manera contextualizada y con pertinencia social, en el desarrollo de sus actividades académicas cotidianas de su formación (GARCÍA; AZNAR, 2019 ) y LÁREZ: Y GUILLÉN, (2020); a través de la identificación de situaciones problematizadas y el diseño e implementación de acciones metodológicas específicas, para resolver situaciones inherentes a su desempeño laboral docente (DE LAS SALAS; MARTÍNEZ, 2011).

En el particular de las ofertas curriculares de los programas de posgrado profesionales no son asociados de manera directa a temas investigativos desde la formación que se esperaría que el estudiante adquiriera en su proceso de académico, de tal modo las habilidades investigativas pasan a un segundo plano en las acciones procedimentales de los saberes curriculares del plan de estudio, es así que la elaboración de trabajos de intervención no se sustentan principalmente desde el conocimiento

Recebido em: 30/05/2021

Aceite em: 13/08/2021 
científico (TURPO-GEBERA; HURTADO-MAZEYRA; DELGADO-SARMIENTO, 2020), así pues la formación curricular del estudiante de posgrado debe estar sustentada por actividades de aprendizaje basado en la solución de problemas que enfrentará al momento de vincularse laboralmente y en adquirir la lengua escrita para la comunicación y divulgación de sus descubrimientos (BIGI et al., 2019).

Las habilidades investigativas y su incorporación en el desarrollo de la praxis profesional docente constituyen en la actualidad, un reto de particular importancia para la formación docente por competencia; en los programas de posgrado de maestría con enfoque profesional. Pues las mismas contribuyen a potenciar las herramientas requeridas por estos profesionales para optimizar los procesos de intervención dirigidos a mejorar la calidad de los procesos gerenciales, curriculares y de convivencia en las instituciones educativas desde una perspectiva científica.

Por lo tanto el objetivo del presente estudio, estuvo orientado a diseñar una propuesta curricular desde un enfoque estratégico (NAVARRO; PEREIRA; PEREIRA; FONSECA, 2010), orientada a fortalecer el desarrollo y consolidación de las principales habilidades investigativas a través de la articulación del eje metodológico en los cursos de Metodología y Seminario de Proyectos de Intervención en Educación y Trabajo Final de Posgrado (TFP) I y II del Magister en Educación de la Universidad Adventista de Chile (UNACH).

\section{METODOLOGÍA}

Este estudio puede tipificarse como una investigación de tipo Proyecto Factible (UPEL, 2016), sustentado en una investigación documental desarrollada bajo un diseño de tipo bibliográfico (CUÉ; ORAMAS, 2008). El corpus de estudio fue seleccionado de manera no probabilística y estuvo conformado por fuentes de tipo bibliográfico y documentos en línea, correspondientes a los años 2005 al 2020 incluidos las mallas curriculares, competencias genéricas, sello y profesionales del programa.

Los criterios utilizados para la selección de los materiales, que conformaron el corpus empleado para dar sustento a la propuesta, fueron la conveniencia y el juicio de los investigadores. Las categorías iniciales utilizadas para guiar la selección de la información fueron las siguientes: (a) habilidades investigativas (b) formación docente Recebido em: 30/05/2021

Aceite em: 13/08/2021 
de posgrado; (c) proyectos de intervención educativa (d) magister profesionalizante y (e) planeación curricular estratégica. La técnica aplicada fue la observación y los instrumentos de recolección de datos fueron las fichas bibliográficas, textuales, resumen y de paráfrasis y la matriz DOFA para el desarrollo del análisis estratégico.

Para el desarrollo de la propuesta curricular se siguió el procedimiento que a continuación se describe: (a) diagnóstico de las habilidades investigativas presentes en el actual perfil de competencias y desarrollo del análisis DOFA a partir de la revisión de los documentos curriculares del Magister en Educación; (b) diseño de la propuesta curricular desde una perspectiva sistémica, con enfoque micro curricular con atención en los resultados del diagnóstico y uso de los elementos estratégicos identificados (c) generación de lineamientos técnico curriculares para la implementación de la propuesta desde el Magister en Educación menciones Currículo y Evaluación, Gestión Liderazgo Educativo y Orientación y Convivencia.

\section{RESULTADOS}

\section{Habilidades investigativas en estudiantes de posgrado profesional}

La construcción del proceso pedagógico en posgrado se puede resumir en que el estudiante del magister en educación pueda adquirir conocimientos y habilidades para lograr ser un especialista en Currículo y Evaluación Educativas, Orientación y Convivencia Escolar o Gerencia Educativa (según la mención seleccionada), aunque dentro del contexto laboral el obtener la especialización es solo la punta del iceberg, si consideramos las constantes problemáticas asociadas a la gestión pedagógica, liderazgo educativo, clima de aula y la gestión de recursos, entre otros que deben ser afrontados por el docente y ser solucionados. En efecto los cambios constantes que ocurren alrededor del conocimiento científico y ordinario afectan a la educación, ya sea, en metodológicas de enseñanza, surgimiento nuevos paradigmas educativos o avances en la investigación, entre otros (GUTIÉRREZ-RICO et al., 2019), esto genera que la realidad educativa y formadora tenga que adaptarse a los nuevos desafíos impuestos por el medio.

Recebido em: 30/05/2021

Aceite em: 13/08/2021 
La transversalidad de las habilidades investigativas es aplicable a varias dimensiones de los procesos de formación de posgrado, indiferentemente si es un programa orientado a la investigación o profesional, la razón de esta vinculación directa radica en el que se entiende como una habilidad los siguientes aspectos a) Búsqueda de información confiable en fuentes primarias y secundarias b) Escritura académica o científica c) Procesamiento de la información de datos cuantitativos y cualitativos d) Gestores bibliográficos e) Normas de citación f) Divulgación del conocimiento académico, las cuales son esenciales para la elaboración de cualquier tipo de trabajo académico o profesional en el área de la educación. El desarrollo de estas habilidades no está exclusivamente asociado a programas investigativos o propios de cátedras asociadas al área de la metodología de la investigación, es por ello que Morales et al (2020) concuerdan en sugerir que las asignaturas que no tributan directamente a la investigación, deben contribuir de igual manera en a la adquisición de habilidades investigativas, un ejemplo de ello es la asignación de actividades evaluativas vinculadas a un modelo orientado por competencias, dentro de los cuales destacan ensayos, seminarios bibliográficos, proyectos de intervención, revisiones bibliográficas y propuestas curriculares o pedagógicas, que se encuentran contenidas en el micro currículo de las asignaturas, por lo tanto no existe un inconveniente curricular, pedagógico o de método de enseñanza en contemplar la transversalidad de la investigación en acciones curriculares de las asignaturas que no pertenecen a la metodología de la investigación (FONTAINES-RUIZ, MAZA-CORDOVA, PIRELA, 2020; KOEPPE, RIBEIRO Y CALABRÓ 2020; GENTIL Y ALCASAR, 2020).

Es necesario mencionar que el constructo de la formación de investigadores en educación nos orienta que el pedagogo sea formado como investigador y transformador de su realidad, así pues, no se presenta una disonancia con lo que se espera que realice un profesional con el grado de magister en educación en su realidad inmediata, debido a que la práctica docente en el aula se entiende como un proceso de intervención pedagógica en el conocimiento empírico-teórico que adquiere el profesor por medio de la praxis educativa y la indagación para la fundamentación del proceso de enseñanza y aprendizaje propio de las ciencias de la educación (CERVANTES, 2019).

Recebido em: 30/05/2021

Aceite em: 13/08/2021 
Sin duda se construyó una barrera dogmática asociada a que las investigaciones objetivas o metodológicamente correctas se confeccionan en el grado académico de doctor o de un magíster asociado a la investigación (TURPO-GEBERA; HURTADOMAZEYRA; DELGADO-SARMIENTO, 2020). Sin embargo, es necesario resaltar que existen orientaciones para vincular un proceso de enseñanza y aprendizaje con la metodología de la investigación, como se ha dicho en el trabajo elaborado por Gouldin y Usherwood (2003) citado por Montesi et al (2017) Es por ello que al referirnos al desarrollar las habilidades investigativas nos orientamos en la formación transversal para la elaboración de soluciones diversas a los problemas educacionales detectados y la difusión del conocimiento producido en los proyectos de intervención.

Sin duda se ha avanzado en los procesos educativos, por la incorporación de cátedras del área de la metodología de la investigación dentro de las mallas curriculares de pregrado vinculada a la pedagogía (CLAURE, 2019), pero aún se mantiene una carencia en la formación instrumental funcional de los futuros egresados, por lo tanto no se aleja de la realidad el promover las habilidades investigativas a nivel de posgrado sin caer en la mirada utópica de categorizar de simplicidad los complementos que brindar el sustento al contenido del método científico para la generación de conocimiento.

La propuesta didáctica que a continuación se presenta, es el resultado del análisis estratégico de la malla curricular del Magister en Educación ofrecido por la Universidad Adventista de Chile en sus menciones Currículo y Evaluación, Gestión Educativa y Orientación y Convivencia, correspondiente al plan de estudio que entró en vigencia en el año 2018 y se mantiene en la actualidad.

\section{Análisis estratégico de los elementos Curriculares asociadas al desarrollo de}

\section{las habilidades investigativas en el Magister en Educación de la Universidad}

\section{Adventista de Chile}

La información que se presenta a continuación, es el resultado del análisis derivado de un conjunto de documentos de orden curricular (malla curricular, plan de estudio y programas de las asignaturas relacionadas con el desarrollo de habilidades investigativas) que han permitido en primera instancia, construir un análisis estratégico a partir de las fortalezas, debilidades, oportunidades y amenazas detectadas en el Recebido em: 30/05/2021

Aceite em: 13/08/2021 
proceso, para generar así la propuesta didáctica que se muestra de forma progresiva a continuación.

Cuadro 1 - Análisis estratégico de los elementos relacionados de manera directa e indirecta con el desarrollo de las habilidades investigativas en el Magister en Educación de la Universidad Adventista de Chile.

\begin{tabular}{|c|c|}
\hline Fortalezas & Oportunidades \\
\hline $\begin{array}{l}\text { - Interés institucional por fortalecer los } \\
\text { Programas de Posgrado. } \\
\text { - Consolidación de la Comisión Curricular } \\
\text { del Magister en Educación (MGE). } \\
\text { - Disposición de los profesores a integrarse } \\
\text { en el proceso. } \\
\text { - Perfil profesional de los miembros del } \\
\text { Comité académico del MGE. } \\
\text { - Adopción del modelo de formación por } \\
\text { competencias en el MGE }\end{array}$ & $\begin{array}{l}\text { - Cercanía del proceso de Acreditación } \\
\text { institucional por la Comisión Nacional } \\
\text { de Acreditación (CNA). } \\
\text { - Condiciones contextuales propicias } \\
\text { para la proyección extrainstitucional e } \\
\text { internacionalización del MGE. } \\
\text { - Demanda creciente de posgrados en } \\
\text { línea en la región }\end{array}$ \\
\hline $\begin{array}{l}\text { Debilidades } \\
\text { - Escasez y desarticulación de contenidos } \\
\text { orientados al desarrollo de habilidades } \\
\text { investigativas en las materias que } \\
\text { conforman el eje de aplicación de la malla } \\
\text { curricular del MGE. } \\
\text { - Resistencia al cambio en algunas } \\
\text { instancias institucionales en relación con la } \\
\text { adopción del enfoque por competencias. } \\
\text { - Necesidad de actualizar y formar al } \\
\text { profesorado para promover el desarrollo de } \\
\text { habilidades de investigación en los } \\
\text { estudios de posgrado }\end{array}$ & $\begin{array}{l}\text { - Existencia de programas similares en } \\
\text { el área de influencia de la UNACH } \\
\text { - Cambios recientes en los criterios de } \\
\text { Acreditación de la CNA para programas } \\
\text { de postgrado } \\
\text {-Inestabilidad política, económica, } \\
\text { social y educativa generada por la } \\
\text { situación de pandemia }\end{array}$ \\
\hline
\end{tabular}

Fuente: Autores, 2021.

Recebido em: 30/05/2021

Aceite em: 13/08/2021 
As Al analizar el cuadro anterior puede observarse que las fortalezas reportadas guardan relación con elementos de particular interés para promover el desarrollo de habilidades investigativas desde el MGE, como lo es el apoyo institucional a los procesos curriculares, la disponibilidad de personal docente capacitado e interesado en participar y la adopción del modelo de formación por competencias en el postgrado de la institución.

Desde la perspectiva de las debilidades, estas guardan relación con la escasez de contenidos en los planes y programas de estudio orientados al desarrollo de las habilidades investigativas, pero también con la preparación y resistencia al cambio presentes en algunos docentes. Situación que sin lugar a duda debe ser atendida para viabilizar el éxito de la propuesta didáctica que se presenta.

Por su parte, las oportunidades presentes en el contexto se perfilan fundamentalmente en torno a la posibilidad de aprovechar los procesos de acreditación con los que debe cumplir próximamente la organización, así como con la posibilidad de aprovechar las demandas regionales referidas a la oferta de estudios en línea para captar posibles participantes. Con respecto a las amenazas, pueden destacarse los elementos referidos a la existencia de otros programas de maestría en la región que pudieran influir negativamente en la captación de participantes, así como los últimos cambios en los criterios de acreditación de los programas de posgrado realizados por la CNA y las condiciones generadas por la actual pandemia en los ámbitos político, económico, social y educativo.

\section{Análisis de la Estructura curricular del eje de aplicación como referente al} desarrollo de las habilidades investigativas en estudiantes de posgrado profesional

De acuerdo con la malla curricular del MGE de la Universidad Adventista de Chile, este programa de maestría profesional se encuentra estructura en un total de 60 Unidades de crédito distribuidas en un total de cuatro periodos académicos y organizadas en función a tres ejes fundamentales los cuales se esbozan brevemente a continuación: (a) formación común, el cual incluye un conjunto de unidades curriculares

Recebido em: 30/05/2021

Aceite em: 13/08/2021 
que son comunes a todos los participantes independientemente de las mención que haya seleccionado; (b) formación avanzada, conformado fundamentalmente por las materias cuyo contenido tributa desde la perspectiva disciplinar al desarrollo de habilidades y destrezas asociadas a la mención específica que cursa el participante y (c) aplicación, correspondientes a las unidades curriculares asociadas al desarrollo de proyectos de intervención y del proyecto final de posgrado. Estas últimas constituyen el tema central de la propuesta didáctica dirigida al desarrollo de las habilidades investigativas aplicadas a la docencia en el MGE de la Universidad Adventista de Chile:

Figura 1 Distribución de los cursos que conforman el eje de aplicación por periodo académico

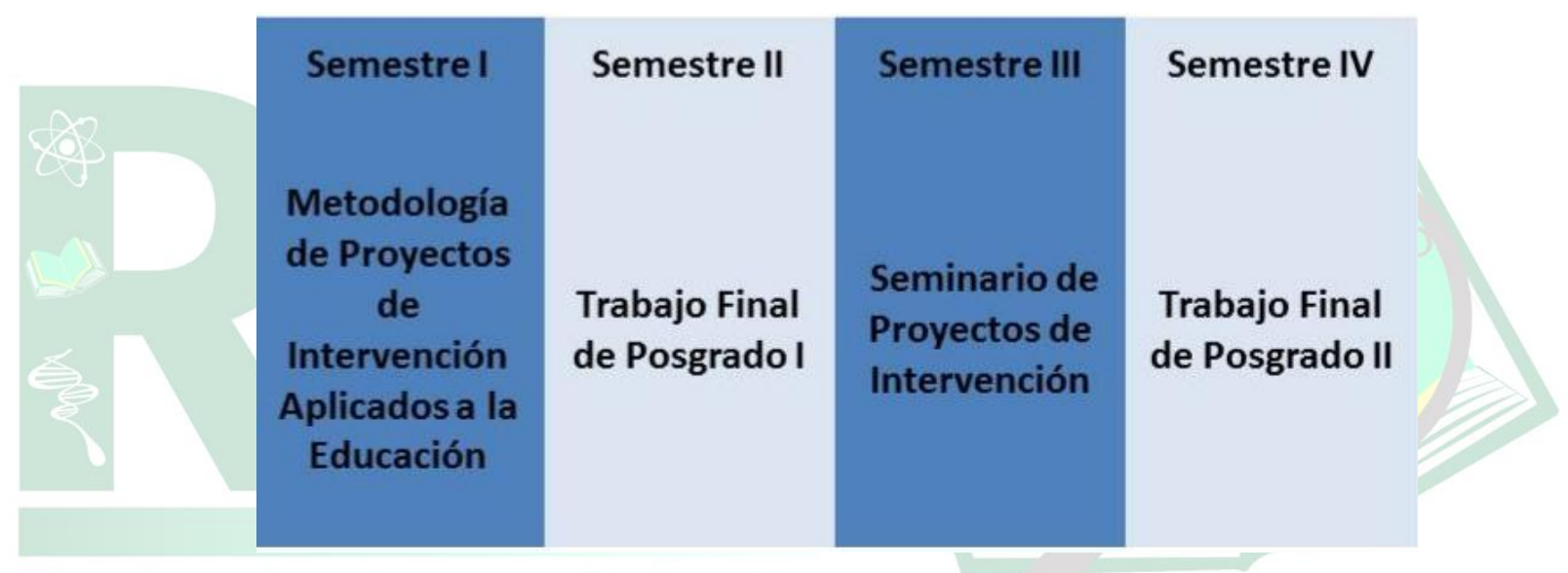

Fuente: Sobarzo-Ruiz; Lárez-Hernández, 2021.

As Como puede observarse en el gráfico anterior, las materias correspondientes al eje de aplicación se encuentran distribuidas a lo largo de los cuatro períodos académicos en que se ha estructurado el MGE. Es importante destacar que por ser un programa de orientación profesional el énfasis como ya se ha mencionado, se encuentra dirigido a la especialización en el área disciplinar correspondiente y a la aplicación de los aprendizajes construidos en la resolución de situaciones inherentes a su ámbito de desempeño a partir del desarrollo de proyectos de intervención educativa.

Si bien es cierto que existen dos materias asociadas con la intervención educativa, como es el caso de la Unidad Curricular Metodología de Proyectos de Intervención Aplicados a la Educación y Seminario de Proyectos de Intervención insertos en el I y III semestre respectivamente y de las UC Trabajo Final de Posgrado I (TFP I) y Trabajo Recebido em: 30/05/2021

Aceite em: 13/08/2021 
Final de Posgrado II (TFP II), en el II y IV semestre respectivamente. La revisión del enfoque y núcleos temáticos existentes en los actuales programas permitieron identificar los siguientes elementos: (a) poco desarrollo de los elementos conceptuales y teóricos referidos al proceso de intervención educativa, persistiendo un enfoque predominantemente práctico en los cursos orientados al desarrollo de habilidades referidas a la intervención educativa; (b) desarticulación entre los contenidos derivados de los núcleos temáticos entre las UC incluidas en el I y III semestre situación que dificulta el avance del estudiante y el seguimiento por parte de los profesores guías asignados como tutores.

Es importante destacar que esta misma situación se notó en el caso de las UC TFP I y TFP II, en las cuales se observa en su administración un enfoque instrumentalista, orientado al desarrollo de actividades de orden práctico, que si bien permiten hasta cierto punto contribuyen al avance del participante, no le proveen de los elementos necesarios para sustentar desde el punto epistemológico su trabajo, con lo que podrían producirse inconsistencias metodológicas en su desarrollo, capaces de afectar la calidad del producto final obtenido.

Propuesta didáctica para promover el desarrollo de habilidades investigativas a partir de la articulación de las Unidades Curriculares que conforman el eje de aplicación en el currículo del Magister en Educación

Es por ello que, atendiendo los planteamientos previamente realizados, se elaboró esta propuesta didáctica, dirigida a enriquecer por una parte los núcleos temáticos a considerar en cada una de las UC que componen el eje de aplicación y por la otra, a articular los procesos de intervención e investigación para promover el desarrollo de habilidades de investigación en los docentes que cursan el MGE:

Cuadro 2 - Propuesta didáctica centrada en la construcción de los núcleos temáticos que permitan la articulación de las unidades curriculares del eje de aplicación de la malla curricular del MGE para promover el desarrollo de habilidades investigativas en los docentes- participantes

\begin{tabular}{|c|c|c|}
\hline Semestre & Unidad & Núcleos temáticos propuestos \\
\hline
\end{tabular}

Recebido em: 30/05/2021

Aceite em: 13/08/2021 
Edição Especial: Pesquisa na Pós-Graduação em Ensino de Ciências

Vol. 4, n. 5. 2021

ISSN: $2595-4520$

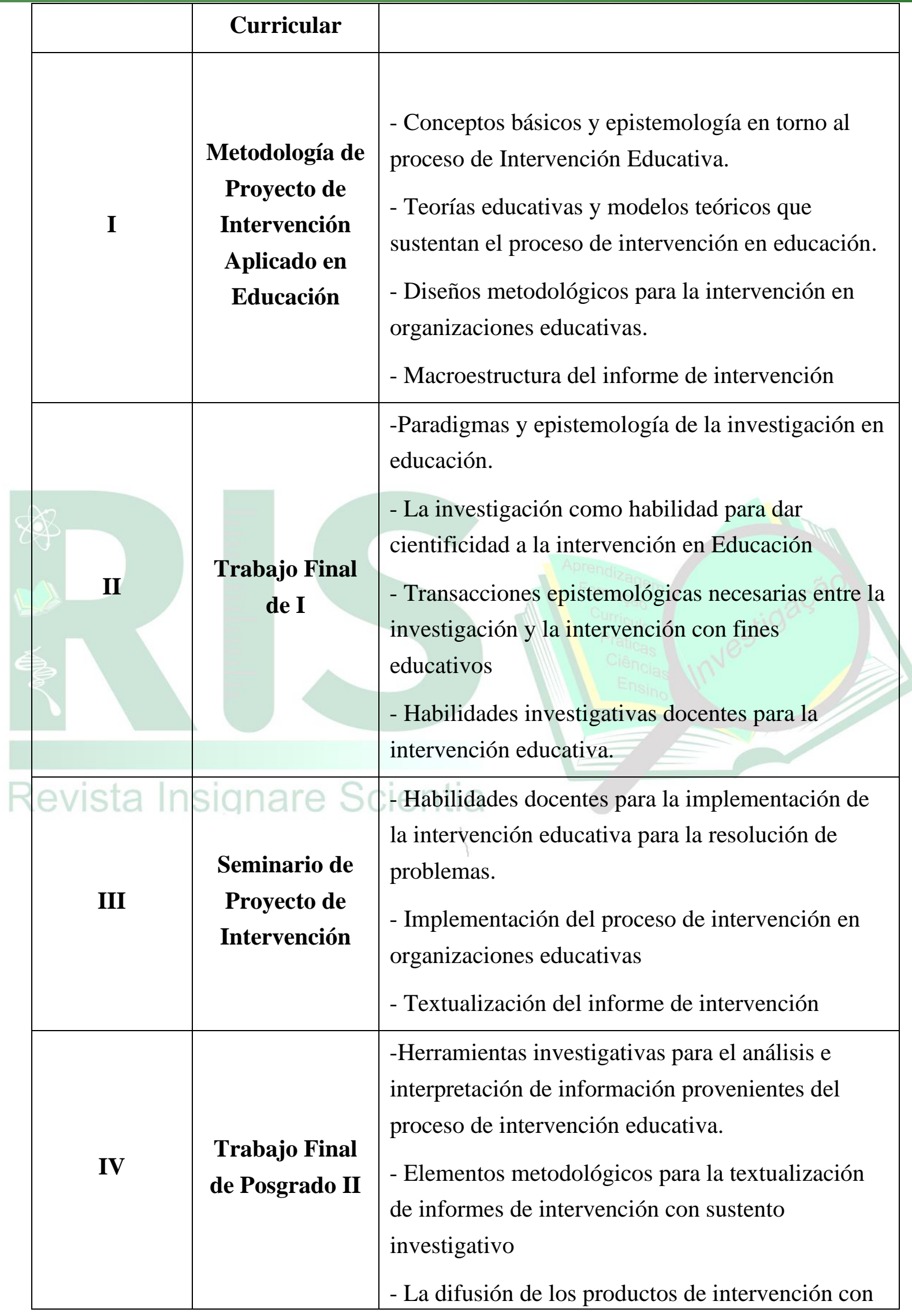

Recebido em: 30/05/2021

Aceite em: 13/08/2021 


\begin{tabular}{|l|l|l|}
\hline & $\begin{array}{l}\text { sustento investigativo como cierre del ciclo de } \\
\text { producción de conocimiento y transformación del } \\
\text { conocimiento tácito en explícito }\end{array}$ \\
\hline
\end{tabular}

Fuente: Autores, 2021.

Como puede observarse en el cuadro anterior, los núcleos temáticos que forman parte de la propuesta didáctica elaborada buscan contribuir con la creación de un espacio de aprendizaje significativo, que se articule a través de las cuatro UC curriculares que, en su conjunto, conforman el eje de aplicación del plan de estudio del MGE de la UNACH. Estas han sido tomadas por los autores del presente estudio, como referente para el desarrollo de las habilidades investigativas, que permitan articular los procesos de intervención e investigación, para dar soluciones eficaces y adecuadas a las realidades existentes en las instituciones educativas donde les corresponderá actuar.

\section{CONCLUSIONES}

El desarrollo de las habilidades investigativas requiere de un enfoque transversal en su abordaje en las unidades micro-curriculares que conforman el eje metodológico del plan de estudio, de tal modo que se permita articular de forma orgánica entre los resultados de aprendizajes y los indicadores de evaluación, permitiendo evidenciar en las instancias evaluativas la adquisición de las habilidades, ya sea en la búsqueda, análisis, síntesis de la información de fuentes primarias o secundarias siendo concretadas en la escritura academia para la construcción de actividades evaluativas vinculantes a micro investigaciones por parte del estudiantado.

El dominio de los elementos investigativos por los participantes del programa es indispensable para garantizar la dimensión científica en los procesos del diseño de los proyectos de intervención aplicados a la educación, logrando generar un sustento empírico y metodológico para el levantamiento de las soluciones a los problemas detectados, de igual manera se abordan los aspectos asociados al análisis de los resultados, de esta manera se obtiene una cientificidad al procesamiento e interpretación de la información. Es así que como, el desarrollo de habilidades para la ubicación, selección, procesamiento y uso de información de calidad en el abordaje de situaciones Recebido em: 30/05/2021

Aceite em: 13/08/2021 
problematizadas, a través de los procesos de intervención es fundamental, para contribuir a consolidar el carácter profesionalizante del magister;

Las habilidades para la textualización del informe de intervención y del Trabajo Final de Posgrado, así como la difusión de los resultados obtenidos, forman parte del perfil de las habilidades investigativas deseables de los estudios de posgrado, independiente si es un programa académico o profesionalizante, así pues, en el medio profesional dichas habilidades son bien valoradas para desempeñar distintas funciones en relación cona la docencia y consultoría en el mundo privado y público. Por lo tanto, tienen un carácter heurístico y deben orientarse a la identificación de situaciones problemáticas, el diseño de proyectos de intervención compatibles con los problemas educativos detectados y el diseño de soluciones con pertinencia social y respeto a los criterios deontológicos que rigen el ejercicio de la profesión docente.

\section{REFERENCIAS}

Bigi, Elisa; García, Marisol; Chacón, Edixon. ¿Qué textos académicos escriben los estudiantes universitarios de Educación?. Zona Próxima, n. 31, p. 25-55, 2019.

Cervantes, Evangelina. Un Acercamiento a la Formación de Docentes como Investigadores Educativos en México. REICE. Revista Iberoamericana Sobre Calidad, Eficacia y Cambio En Educación, Vol 17, n. 4, pág 59-74, 2019.

Claure, José. Modelo didáctico para la enseñanza de la metodología de la investigación científica. Gaceta Médica Boliviana, Vol 42, n. 2, pág 199-201, 2019.

Cué, Manuel; Oramas, Jehová. Síntesis de información y artículos de revisión. Revista ACIMED, Vol 17, n. 2, pág1-11, 2008.

De las Salas, Magdy; Martínez, César. Competencias técnicas investigativas en los docentes del núcleo LUZ - Costa Oriental del Lago. Telos, v. 13, n. 3, p. 412-429, 2011.

Fontaines-Ruiz, Tomás; Maza-Cordova, Johann; Pirela, Jorge. Memoria del Congreso Virtual Iberoamericano sobre Tendencias en Investigación: Metodologías, alfabetizaciones, formación y comunicación científica. Primera edición. RISEI, 2020.

García-Gutiérrez Zaily; Aznar-Díaz, Inmaculada. El Desarrollo de competencias investigativas, una alternativa para formar profesionales en pedagogía infantil como personal docente investigador. Revista Electrónica Educare, v. 23, n. 1, p. 1-22, 2019.

Gentil, Marcia; Alcasar, Leandro. La investigación como principio pedagógico: los desafíos de la praxis docente para la resignificación del conocimiento. Revista

Insignare Scientia - RIS , v. 3, n. 3, p. 224 - 240, 2020.

Recebido em: 30/05/2021

Aceite em: 13/08/2021 
Gutiérrez-Rico, Dolores; Almaraz-Rodríguez, Omar; Bocanegra-Vergara,

Netzahualcóyotl. Concepciones del docente en sus formas de percibir el ejercicio de la investigación desde su práctica. Revista De Investigación, Desarrollo E Innovación, v. 10, n. 1, p 149-161, 2019.

Koeppe, Cleise; Ribeiro, Marcus; Calabró, Luciana. Para una docencia investigadora: concepciones de los profesores sobre la investigación como actitud y como estrategia pedagógica. Revista Insignare Scientia - RIS, v. 3, n. 3, p. 64-83, 2020.

Lárez, José; Guillén, Gustavo. Perfil de competencias Investigativas de Interés para la Formación Inicial del Profesorado de Ciencias: Una Visión Desde la Práctica Profesional. Encuentro Entre Líneas. Caracas: Subdirección de Investigación y Postgrado. Coordinación General de Investigación, 2019.

Montesi, Michela; Cuevas-Cervero, Aurora; Fernández-Bajón, María. Enseñanza de la metodología de la investigación en ciencias sociales: el punto de vista del alumno de máster. Transinformação, v. 29, n. 3, p 333-342, 2017.

Morales, Héctor et al. Tareas docentes para desarrollar habilidades investigativas desde la asignatura Metodología de la Investigación. Edumecentro, v. 12, n. 1, p. 131-150, 2020.

Navarro, Yasmile; Pereira, Morela; Pereira, Lilia; Fonseca, Neimar. (2010). Una Mirada a la Planificación Estratégica Curricular. Revista de Estudios Interdisciplinarios en Ciencias Sociales, v. 12, n. 2, p. 202-216, 2010.

Turpo-Gebera, Osbaldo; Hurtado-Mazeyra, Alejandra; Delgado-Sarmiento, Yvan; Mango, Pedro. (2020). Formación de investigadores en educación : entre la performatividad y el credencialismo. Revista ESPACIOS, v. 21, n. 4, 2020.

Universidad Pedagógica Experimental Libertador. Manual de Trabajos de Grado de Especialización y Maestría y Tesis Doctorales. Caracas: Vicerrectorado de Investigación y Postgrado, 2016.

Recebido em: 30/05/2021

Aceite em: 13/08/2021 